\title{
Radial Variation of Refractive Index, Plasma Frequency and Phase Velocity in Laser Induced Air Plasma
}

\author{
Manny Mathuthu, Member, IEEE, Rita M. Raseleka, Andrew Forbes, and Nicholas West
}

\begin{abstract}
Laser-induced breakdown spectroscopy (LIBS) is a nonintrusive technique that needs no sample preparation and even recently, quantitative measurements were done without the need for calibration standards. Much research has been done on the laser induced air plasma to study the spatial variation of plasma parameters in the axial direction of the laser beam. In this paper, we report investigation on the radial variation of the refractive index, plasma frequency, and phase velocity of a plasma during laser induced breakdown of air. The results show that the radial variations of the plasma frequency and refractive index are caused by the radial electron density gradient of the plasma. The radial variation of the electron density shows a noticeable depletion at the center of the focus which results in plasma phase velocities greater than the speed of light. Further from the plasma core the electron density diminishes to ambient density at the edges of the plasma. The mean full-width at half maximum (FWHM) for these plasma profiles was found to be about $1.2 \mathrm{~mm}$. The results further reveal that the plasma has a concave parabollic electron density and a convex parabollic refractive index profile near the center of the laser axis, i.e., around a diameter of $0.5 \mathrm{~mm}$.
\end{abstract}

Index Terms-Laser-induced breakdown spectroscopy (LIBS), phase velocity, plasma frequency, refractive index.

\section{INTRODUCTION}

A IR breakdown will occur when the electron density is equal to or greater than a critical threshold. In laserinduced breakdown spectroscopy (LIBS), free electrons collide with neutral atoms and absorb energy from the laser resulting in gas ionization. This inverse Bremsstrahlung process dominates other gas ionization processes [1]. The first stage is breakdown as the gas is ionized, followed by laser energy absorption by the hot gas, and the last stage is detonation which results in plasma expansion and shock wave formation [2], [3]. The evolution of the plasma and the resulting shock waves have been studied both experimentally and theoretically [2], [4]-[6].

LIBS has proven itself to be one of the most versatile tools in plasma diagnosis. However, its greatest limitation has always been the sample matrix effect [7] or the type of material being

Manuscript received January 27, 2006; revised August 9, 2006. This work was supported by the African Laser Center (ALC).

M. Mathuthu is with the Physics Department, University of Zimbabwe, Mt. Pleasant, Harare, Zimbabwe (e-mail: mathuthu@ science.uz.ac.zw).

R. M. Raseleka is with the Tshwane University of Technology, Pretoria 0001, South Africa.

A. Forbes is with the CSIR-National Laser Centre, Pretoria 0001, South Africa and also with the School of Physics, University of Kwazulu-Natal, Durban 4000, South Africa.

$\mathrm{N}$. West is with the Electrical and Information Engineering Department, University of Witswatersrand, Wits, Johannesburg 2050, South Africa.

Digital Object Identifier 10.1109/TPS.2006.887769 analyzed [8]. There was always a need for a different calibration curve for each sample matrix in which the element being analyzed was contained. The intensity of the spectral signal of an element depends on the matrix (solid, liquid, slurry, vapor, or gas) in which it is contained at the time of analysis by LIBS. Apart from that, LIBS is nonintrusive, needs no sample preparation, and can be used to detect multielements in one scan.

The LIBS technique has found vast applications as a diagnostic tool in elemental composition analysis for trace pollutants in soil [9], in water [10], and for studying particle velocity, electron temperature, and density in air plasmas [6], [11]. This versatility enables the LIBS technique to compete with other diagnostic techniques such as: XRF, AAS, ICP-OES, interferometry, and beam deflectometry [5]. In LIBS, the quantitative infomation can be obtained on the entire sample composition in a single run. Just recently, LIBS has been given a shot in the arm when it was shown that it can be used calibration free [7], [12], [13].

A comprehensive overview of the documented research (theoretical and experimental) is given in [14]. Here, parameters such as laser power, wavelength, and pulse width [full-width at half maximum (FWHM)], ambient gas choice, detector optimization, etc., are reviewed, giving the new LIBS user an aggregated reference bank.

However, most of the work previously referred to did not look at the effect of the laser energy on the refractive index of the generated plasma. Also, to our knowledge, there is not enough research on the radial profiles of electron temperature, density, plasma frequency, refractive index, and phase velocity. However, axial profiles of some of these parameters have received considerable attention [1], [5] and for air, an attempt has been made to study the shock and thermal waves using beam deflection techniques [15]. Here, the plasma was shown to suffer changes in refractive index by deflecting a He-Ne beam passing through it. This was explained to be caused by the plasma effects overlapping with the shock waves. The published results show that the plasma expands in the axial direction, preferentially towards the focusing lens. Species with higher ionization states have been shown to propagate faster than those with less ionized states [5].

A study of plasma spherical shock waves using holographic techniques [3] showed that electron and neutral density pertubations cause phase changes between the two exposure beams. The hollographic 3-D fringe pattern observed showed that the shock wave expanded to a radius of $6 \mathrm{~mm}$ in $3 \mu \mathrm{s}$ in air while the electron density decreased from $3.8 \times 10^{19} \mathrm{~cm}^{-3}$ at $r=0$ to zero at $r=1.5 \mathrm{~mm}$ within $650 \mathrm{~ns}$. 
This work reports an investigation on the radial variation of the refractive index, plasma frequency, electron density, and phase velocity changes of an air plasma generated by laser-induced breakdown of air. Some deductions and comparison with theoretical results previously discussed are also presented. Measuring the refractive index quantitatively gives an indication of how much the plasma phase velocity increases above the speed of light in a laser induced plasma. These parameters are functions of plasma frequency which, in turn, is a fuction of plasma electron density. Such information is valuable in experiments aimed at harnessing (controlling) lightning discharges via laser induced plasmas. In Section II, the theoretical backgroud is presented, while Section III describes the experimental setup and procedure for the work reported here. Results and discussions on electron density, plasma frequency, phase velocity changes, and other possible applications of the LIBS technique are covered in Section IV. Finally, Section V presents the conclusions derived from this work.

\section{THEORY}

\section{A. Temperature Measurement}

Assuming that the plasma is in local thermodynamic equilibrium (LTE), then the Boltzman distribution can be used to estimate the population of an excited state. The measured emission intensity $\left(I_{\mathrm{nm}}\right)$ of a line is related to the population of the excited state by the following equation [5], [7]:

$$
I_{\mathrm{nm}}=F C_{s} A_{\mathrm{nm}}\left(\frac{g_{m}}{Z \lambda_{\mathrm{nm}}}\right) \exp \left(-E_{m} / k_{B} T_{e}\right)
$$

where $C_{s}$ is the concentration of the emitting species, $A_{\mathrm{nm}}$ is the transition probability, $\lambda_{\mathrm{nm}}$ is the emitted wavelength of the detected line, $g_{m}$ is the statistical weight of the upper level of the transition, $E_{m}$ is the excitation energy, $k_{B}$ is the Boltzmann constant, and $Z$ is the partition function of the emitting specie at the electron temperature $T_{e}$. The collecting optical system affects the intensity of the detected signal so that the actual measured signal differs from the original emission signal. Thus, in (1), $F$ is the experimental parameter that accounts for the optical efficiency of the collecting system, plasma density, and volume [12]. Now, we define the following quantities:

$$
\begin{aligned}
y & =\ln \left(\frac{I_{\mathrm{nm}} \lambda_{\mathrm{nm}}}{g_{m} A_{\mathrm{nm}}}\right) \\
x & =E_{m} \\
m & =-\frac{1}{K_{B} T} \\
c_{s} & =\ln \left(\frac{C_{s} F}{Z(T)}\right)
\end{aligned}
$$

and substituting (2) into the natural logarithms of (1) gives the following equation that defines the Boltzmann plane:

$$
y=m x+c_{s} .
$$

For each species in a sample, the slope $m$ gives the plasma temperature while the intercept $\left(c_{s}\right)$ gives the sample concentration.

\section{B. Density Measurement}

The collisional processes between atoms and ions affect the shape and width of the spectral line. One such process is the Stark broadening due to collisions of charged species. Not only is the line broadened, but the peak wavelength is shifted. The electron density in such collisions is given by the Saha-Boltzman equation [7], [16]

$$
N_{e}=6.0 \times 10^{21}\left(\frac{g_{o}^{I I} T_{e}^{3 / 2}}{g_{o}^{I}}\right) \exp \left(\frac{-E_{N I}}{T_{e}}\right) \frac{N I}{N I I}
$$

where the $g_{o}$ refer to the statistical weights of the species of population $N I$ and $N I I$ in their ground state, $E_{N I}$ is the ionization potential of the $N I$ specie in its ground state and $N_{e}$ is the electron density. For the $N I I$ line at $500.325 \mathrm{~nm}$, the ionization potential was obtained from data books as $14.534 \mathrm{eV} \mathrm{[17].}$

The electron temperature and density from (3) and (4), respectively, are then used to get the plasma frequency and the real part of the refractive index for an air plasma.

The plasma frequency $\left(w_{p}\right)$ relates to the electron density according to [18] NII

$$
\omega_{p}^{2}=\frac{N_{e} e^{2}}{m_{e} \epsilon_{o}}
$$

where $\epsilon_{o}$ is the permitivity of free space, $m_{e}$ is the electron mass, and $e$ is the electron charge.

The refractive index is a function of $\omega_{p}$ and laser frequency $(\omega)$. However, the refractive index consists of the imaginary part $\left(n_{i}\right)$ responsible for laser energy attenuation and the real part $\left(n_{r}\right)$ responsible for laser phase change. Their relations to the plasma frequency [19] are as follows:

$$
\begin{aligned}
& n_{r}=\left\{\frac{1}{2}\left(1-\frac{\omega_{p}^{2}}{\omega^{2}+v_{\mathrm{ec}}^{2}}\right)+\frac{1}{2}\left[\left(1-\frac{\omega_{p}^{2}}{\omega^{2}+v_{\mathrm{ec}}^{2}}\right)^{2}\right.\right. \\
& \left.\left.+\frac{v_{\mathrm{ec}}^{2}}{\omega^{2}}\left(\frac{\omega_{p}^{2}}{\omega^{2}+v_{\mathrm{ec}}^{2}}\right)^{2}\right]^{1 / 2}\right\}^{1 / 2} \\
& n_{i}=\left\{-\frac{1}{2}\left(1-\frac{\omega_{p}^{2}}{\omega^{2}+v_{\mathrm{ec}}^{2}}\right)+\frac{1}{2}\left[\left(1-\frac{\omega_{p}^{2}}{\omega^{2}+v_{\mathrm{ec}}^{2}}\right)^{2}\right.\right. \\
& \left.\left.+\frac{v_{\mathrm{ec}}^{2}}{\omega^{2}}\left(\frac{\omega_{p}^{2}}{\omega^{2}+v_{\mathrm{ec}}^{2}}\right)^{2}\right]^{1 / 2}\right\}^{1 / 2}(6)
\end{aligned}
$$

where

$$
v_{\mathrm{ec}}=\frac{e^{4} N_{e}}{\epsilon_{o}^{2} \sqrt{m_{e} T_{e}^{3}}}
$$

is the electron-neutral collision frequency [22].

The laser phase and group velocities [23] are given by

$$
v_{p}=\frac{w}{k}=c\left(1-\frac{\omega_{p}^{2}}{\omega^{2}}\right)^{-1 / 2}, \quad v_{g}=\frac{d w}{d k}=c\left(1-\frac{\omega_{p}^{2}}{\omega^{2}}\right)^{1 / 2} .
$$




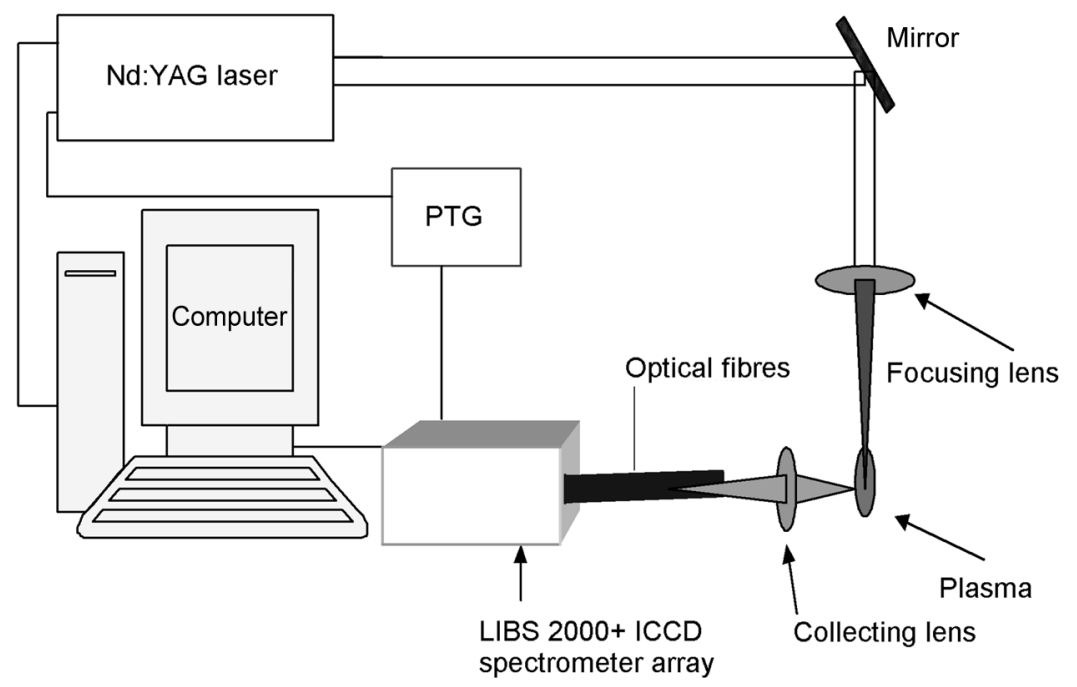

Fig. 1. Schematic of the experimental set-up.

\section{EXPERIMENTAL SETUP AND PROCEDURE}

The schematic setup of the experiment is shown in Fig. 1. The 1064-nm Nd:YAG Q-switched laser (CONTINUUM Powerlite, Model PL9010) has a pulse width of $10 \mathrm{~ns}$ and variable repetition rate from 0 to $10 \mathrm{~Hz}$. The maximum output energy was $1 \mathrm{~J}$ per pulse. The laser energy was measured using an energy meter (SCIENTECH, Model P50). The laser pulse was focused in atmospheric air using a planoconvex quartz lens $(f=150 \mathrm{~mm})$. The maximum spot radius was calculated to be $13.55 \mu \mathrm{m}$ and for a minimum input laser energy of $50 \mathrm{~mJ}$, the minimum intensity was calculated to be $8.7 \times 10^{12} \mathrm{Wcm}^{-2}$. This was above the air breakdown threshold intensity of $2.0 \times 10^{12} \mathrm{Wcm}^{-2}$ as given in [1], [4], and [14]. The detection system was an Ocean Optics LIBS2000+ Spectrometer. It consisted of seven HR2000 high-resolution miniature fiber optic spectrometers. Each has a 2048-element linear charge-coupled device (CCD) array and all the spectrometers are triggered to acquire and read out data simulteneously. The detectors covered the spectral region from UV, VIS, to IR, i.e., from 200 to $980 \mathrm{~nm}$. The LIBS signal was collected by a bundle of seven optical fibers which supply each of the miniature spectrometers. The input ends of the fibers are merged into a single fiber with seven inlets. For this reason, a diffuse piece of glass (sand-blasted quartz) was used in front of the fiber inlet to scatter the light equally to all the seven inlets. The plasma plume was imaged one-to-one onto the entrance of the fiber via a lens system as shown in Fig. 1. The imaging lens and collecting fiber are fixed together on the same stand. A small $x$-translation stage was used to vary the position of the focusing lens. This varied the radial position of the plume relative to the imaging lens system. This method of radial imaging was chosen instead of changing the radial position of the collecting lens relative to the plume, because it was important to keep the optical efficiency of the collecting system constant [7], [20]. The maximum radial translation of the stage from the mean $r=0$ position was $2 \mathrm{~mm}$. Beyond this, the intensity of the signal was too weak. The error introduced by the high quality plano-convex focusing lens when the laser falls on slightly different positions was negligible. The laser pulse delay was synchronized to the
LIBS 2000+ spectrum analyzer using a programmable timing generator (PTG) from Stanford Research Systems (Model DG 535). The delay time between the laser pulse and the signal collecting system was controlled by the Ocean Optics Software that came with the detector. A delay time of $500 \mathrm{~ns}$ was found to give the best signal-to-noise ratio (SNR).

The emission spectrum was averaged over ten scans and stored onto a computer using the Ocean Optics Software. The same software allowed for element identification (using an inbuilt library), peak analysis, FWHM determination, as well as producing time, resolved 2- and 3-D plots.

The Boltzmann plot technique [5] was used to determine the temperature of the plasma at each radial position of the focusing lens. For a multielement sample, multiple parallel lines can be plotted for each element species in the sample. A plot of $\ln \left(I_{\mathrm{nm}} \lambda_{\mathrm{nm}} / g_{m} A_{\mathrm{nm}}\right)$ versus energy $E_{m}$ gives a straight line of negative slope and using (3), the temperature is deduced from this slope. It was clear that the temperature will be the same whichever specie was selected. The NII specie was chosen because it gave the widest energy difference. A linear fit to the experimental data validated the assumption of Maxwellian electron distribution and also served as a check for errors in line assignment and transition probability values.

The NII, 500.325-nm line was also used for determining the electron density because it is one of the strongest lines which could be resolved by our spectrometer. Equation (4) was then used to calculate $N_{e}$ from measured temperatures. This was repeated for each radial position from which plasma parameter profiles were plotted as shown later.

The experiment was repeated for different laser energies to see the effect of laser energy, from threshold energy density to $200 \mathrm{~mJ}$ per pulse.

\section{RESULTS AND Discussions}

Table I shows the lines and other constants used to determine the excitation temperature for air spark by the Boltzmann plot technique. 
TABLE I

SPECTROSCOPIC DATA FOR $N I I$ LINES $^{1}$

\begin{tabular}{lllll}
\hline $\begin{array}{l}\text { Wavelength, } \lambda_{n m}( \pm 0.1) \\
(\mathrm{nm})\end{array}$ & $\begin{array}{l}E_{m} \text { (upper level) } \\
(\mathrm{eV})\end{array}$ & $\begin{array}{l}g_{m} \\
\text { (upper level) }\end{array}$ & $\begin{array}{l}A_{n m} \\
\left(10^{8} s^{-1}\right)\end{array}$ & Configuration \\
399.5 & 21.60 & 5 & 1.35 & $2 \mathrm{~s} 2.2 \mathrm{p} .\left(2 \mathrm{P}^{*}\right) .3 \mathrm{~s}-2 \mathrm{~s} 2.2 \mathrm{p} .\left(2 \mathrm{P}^{*}\right) .3 \mathrm{p}$ \\
460.7 & 21.15 & 3 & 3.26 & $2 \mathrm{~s} 2.2 \mathrm{p} .\left(2 \mathrm{P}^{*}\right) .3 \mathrm{~s}-2 \mathrm{~s} 2.2 \mathrm{p} .\left(2 \mathrm{P}^{*}\right) .3 \mathrm{p}$ \\
500.1 & 23.13 & 7 & 1.05 & $2 \mathrm{~s} 2.2 \mathrm{p} .\left(2 \mathrm{P}^{*}\right) .3 \mathrm{p}-2 \mathrm{~s} 2.2 \mathrm{p} .\left(2 \mathrm{P}^{*}\right) .3 \mathrm{~d}$ \\
500.3 & 20.94 & 3 & 0.08 & $2 \mathrm{~s} 2.2 \mathrm{p} .\left(2 \mathrm{P}^{*}\right) .3 \mathrm{~s}-2 \mathrm{~s} 2.2 \mathrm{p} .\left(2 \mathrm{P}^{*}\right) .3 \mathrm{p}$ \\
500.5 & 23.14 & 9 & 1.16 & $2 \mathrm{~s} 2.2 \mathrm{p} .\left(2 \mathrm{P}^{*}\right) .3 \mathrm{p}-2 \mathrm{~s} 2.2 \mathrm{p} .\left(2 \mathrm{P}^{*}\right) .3 \mathrm{~d}$ \\
566.7 & 20.65 & 5 & 0.37 & $2 \mathrm{~s} 2.2 \mathrm{p} .\left(2 \mathrm{P}^{*}\right) .3 \mathrm{~s}-2 \mathrm{~s} 2.2 \mathrm{p} .\left(2 \mathrm{P}^{*}\right) .3 \mathrm{p}$ \\
568.0 & 20.67 & 7 & 0.53 & $2 \mathrm{~s} 2.2 \mathrm{p} .\left(2 \mathrm{P}^{*}\right) .3 \mathrm{~s}-2 \mathrm{~s} 2.2 \mathrm{p} .\left(2 \mathrm{P}^{*}\right) .3 \mathrm{p}$ \\
568.6 & 20.65 & 3 & 0.19 & $2 \mathrm{~s} 2.2 \mathrm{p} .\left(2 \mathrm{P}^{*}\right) .3 \mathrm{~s}-2 \mathrm{~s} 2.2 \mathrm{p} .\left(2 \mathrm{P}^{*}\right) .3 \mathrm{p}$ \\
\hline
\end{tabular}

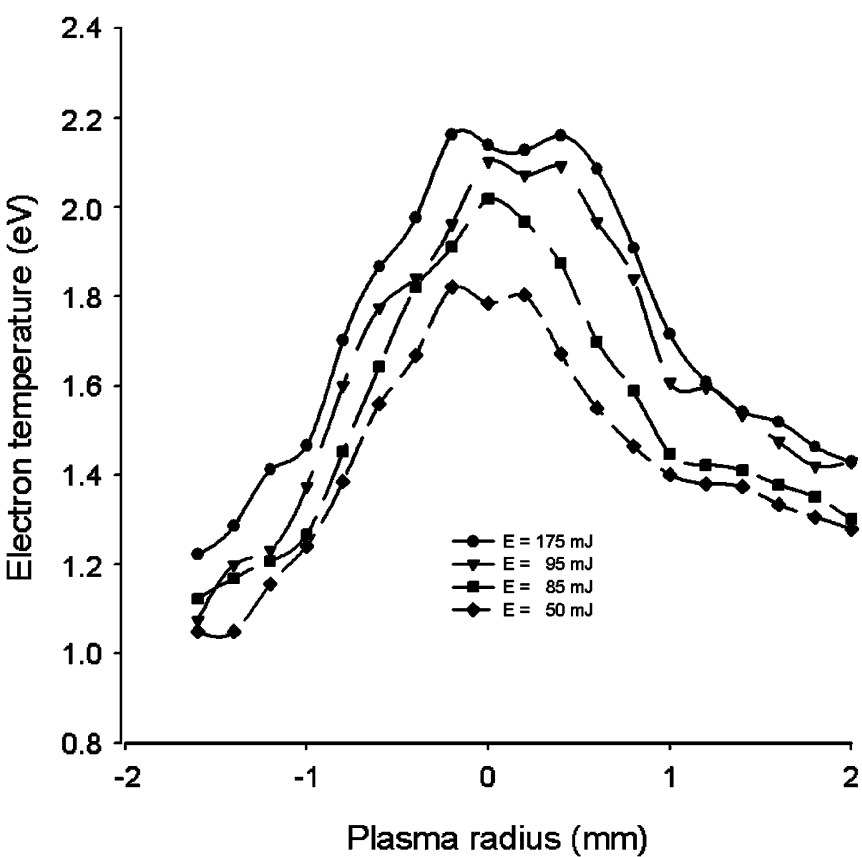

Fig. 2. Variation of electron temperature with plasma radius.

\section{A. Electron Temperature and Density}

Maximum temperature measured for the air plasma was $2.2 \mathrm{eV}$, and the corresponding density was determined to be $7 \times 10^{17} \mathrm{~cm}^{-3}$ for laser energy of $175 \mathrm{~mJ}$. These results are in good agreement with those found in [1], where the energy for breakdown in air was around $200 \mathrm{~mJ}$. LTE was tested using the highest energy gap in the NII lines and was found to give an electron density of $1.87 \times 10^{15} \mathrm{~cm}^{-3}$, as also shown in [11]. The minimum electron density recorded in this work was $1.2 \times 10^{17} \mathrm{~cm}^{-3}$ at the minimum laser energy, which is above the LTE criteria. For the temperature variation with plasma radius, an average of ten scans were used to calculate the temperature from the recorded intensity. Time-integrated intensities were used, therefore, the temperature values presented are indicative of the average conditions in the spark, and not defining the conditions at a particular stage in the plasma. Cutting across the cross section of the plasma shows the strong dependence of the temperature on radial position. From Fig. 2, we see that there are sharp temperature increases within a plasma diameter of $2.0 \mathrm{~mm}$. Also, the higher the energy of the laser, the higher the temperature.

${ }^{1}$ NIST Data Base. [Online]. Available: http://physics.nist.gov/cgi-bin/ASD/ lines.pl

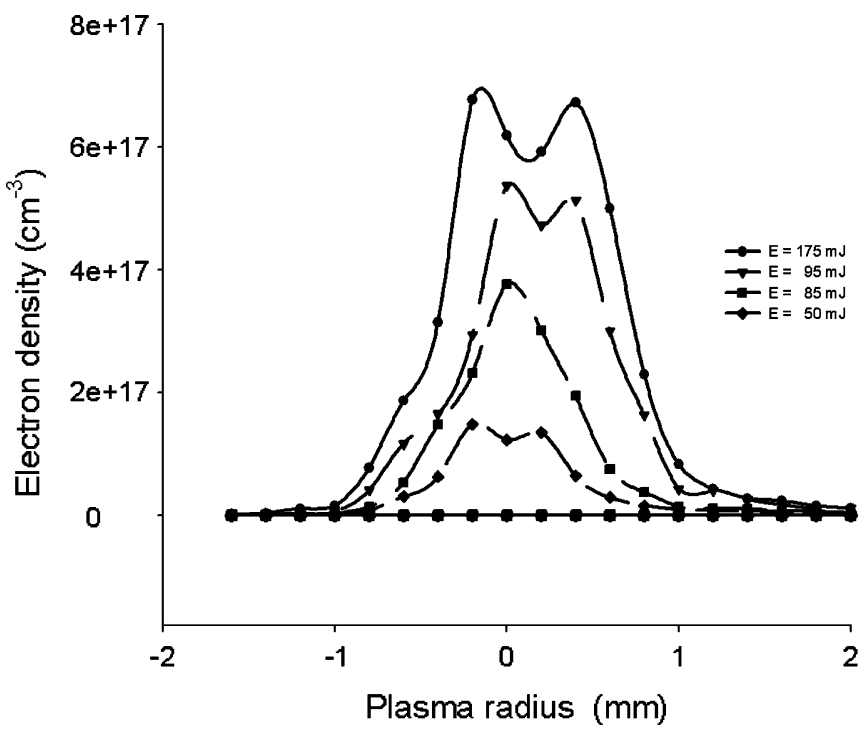

Fig. 3. Variation of electron density versus plasma radius.

However, this increase in plasma temperature with energy is by a factor of less than two. At the edges of the plasma, the temperature approximates the ambient gas temperature. The FWHM of the temperature profile for the largest energy is about $1.8 \mathrm{~mm}$. It can be noticed that the electron temperature difference for the maximum and minimum laser energy used in this work is less than $0.4 \mathrm{eV}$ when the energy difference is as large as $125 \mathrm{~mJ}$ per pulse. This shows that the plasma does not get hotter as it is heated, but it expands [1]. This phenomenon explains the slight deep in plasma temperature observed for laser energies greater than $50 \mathrm{~mJ}$.

Figs. 3-5, are plots of electron density, normalized plasma frequency, and refractive index (real) versus radial distance from the focal point for different laser energies. The choice of negative position is arbitrary and as such, distances to the left of the focal point were chosen to be negative.

Time integrated intensities were used to calculate the electron density, and the values presented here are indicative of the average conditions in the spark, and do not define the conditions at a particular stage in the plasma evolution. Therefore, the results are a mean value out of ten scans. The cross section of the plasma shows the strong dependence of the density on radial position. In Fig. 3, the electron density for the highest input energy $(E=175 \mathrm{~mJ})$ has a FWHM of about $1.2 \mathrm{~mm}$. The plasma had a slight hollow at the center where the electron density was less than the immediate surroundings. That is, the density had a concave parabollic shape as also observed in [21], within a 


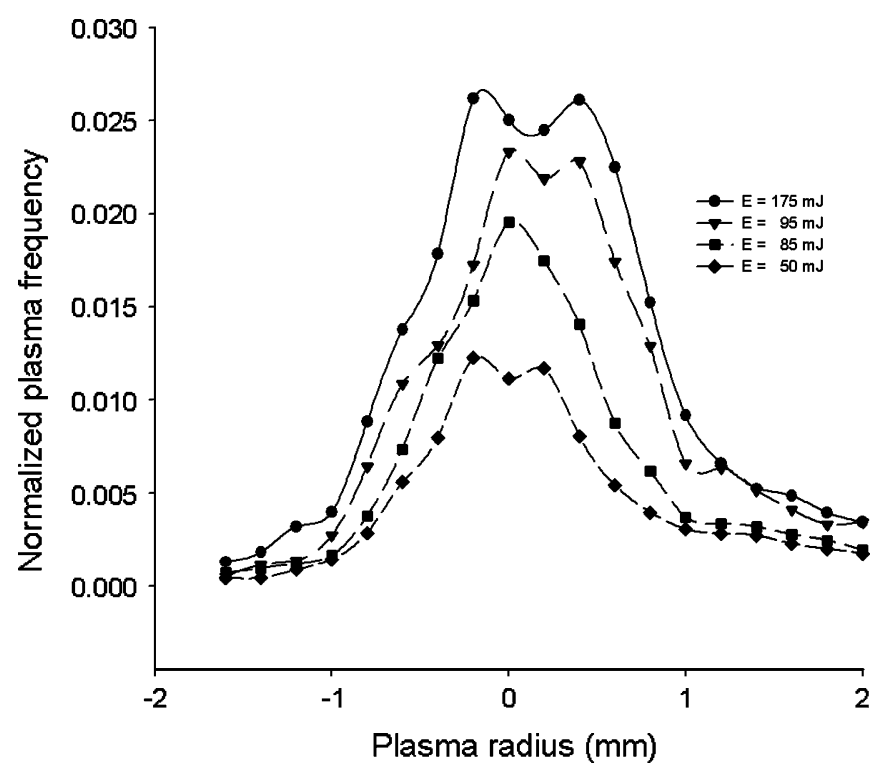

Fig. 4. Variation of normalized plasma frequency versus plasma radius.

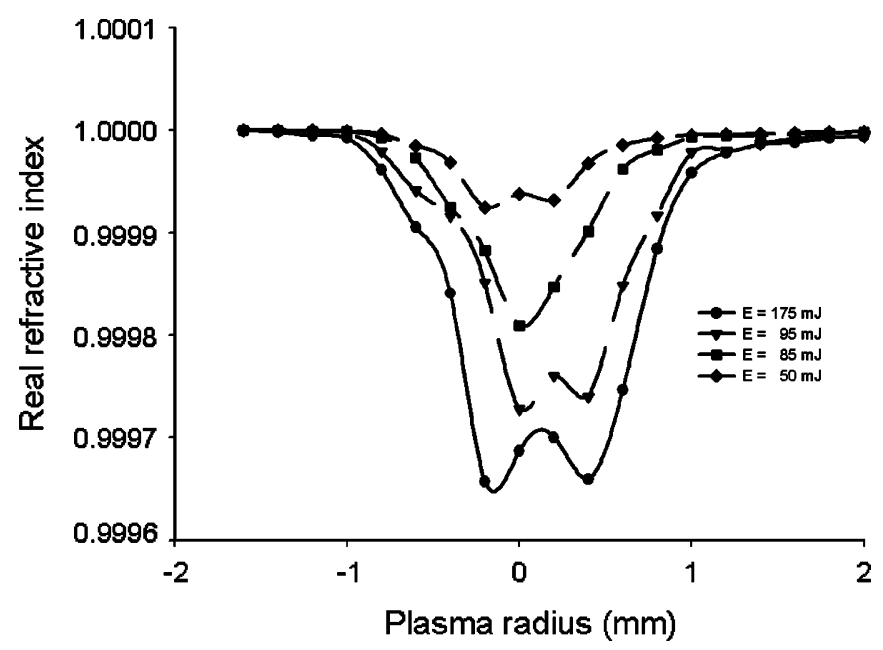

Fig. 5. Variation of real part of refractive index versus plasma radius.

plasma diameter of $0.5 \mathrm{~mm}$ around the axis. The slight hollow in the plasma could be due to a shock wave which blows the free electrons and compresses them against the cold neutral gas at the boundary [4]. This creates a slight void at the center of the plasma, while at the same time, cooling the plasma. The results presented here are in good agreement with the numerical results by Zeng et al. [2], where they considered only the positive radial variation of electron density, and also agree with the experimental results by Gatti et al. [3]. The interferograms in [3] also show the void at the center of the plasma spark. It is important to note that in these two references and this paper, the density also depends on the time after the laser pulse. For this paper, the scan delay time was about $500 \mathrm{~ns}$.

\section{B. Plasma Frequency and Laser Phase Velocity Change}

Since laser energy absorption in a plasma depends on whether the plasma frequency $\left(\omega_{p}\right)$ is less than the laser frequency $(\omega)$, the plasma frequency versus plasma radius for different laser energies was plotted. Fig. 4 shows that in this paper the normal- ized plasma frequency was always less than the laser frequency, which was calculated to be $\omega=1.77 \times 10^{15} \mathrm{~s}^{-1}$. The maximum electron-neutral collision frequency was calculated to be $v_{\mathrm{ec}}=2.09 \times 10^{10} \mathrm{~s}^{-1}$. The normalized plasma frequency for the highest input laser energy has a FWHM of about $1.3 \mathrm{~mm}$. One can, thus, deduce that for the air plasma in this paper, there exists an evanescent domain defined by $v_{\mathrm{ec}}=\leq \omega_{p} \leq \omega$, where laser energy is absorbed in the plasma. The laser energy is not cutoff in the plasma since $\omega_{p} \leq \omega$; a certain percentage is still transmitted through the plasma. The plasma frequency has the same profiles as the electron density in good agreement with (5).

The effect of the laser energy on the refractive index of an air plasma was investigated for energies from 50 to $200 \mathrm{~mJ}$ per pulse. Now, refractive index is a function of plasma frequency and electron collision frequency. It has a real part, which is responsible for laser phase change and an imaginary part, responsible for laser energy attenuation. Only the variation of the real part of the refractive index with plasma radius, as shown in Fig. 5, is reported here. These results show that, within the laser energies used in this paper, the refractive index decreases and the laser velocity in the plasma increases above the velocity of light in vacuum. The plasma phase velocity becomes greater than the velocity of light. This is indeed so, since it is the phase velocity which changes in the plasma. Information and energy travel at the laser group velocity and this cannot be greater than the speed of light. Our results show that for the laser energies used, and within a plasma diameter of $0.5 \mathrm{~mm}$, the plasma has a convex parabollic refractive index. This configuration of the refractive index and density in the plasma makes a plasma capable of guiding a Gaussian beam without much distortion and independent of wavelength [21]. The laser defocuses itself reducing the effect of the focusing lens. Thus, the plasma-laser system enters into a self-regulating regime of self-focusing and defocusing [1], where the plasma limits catastrophic breakdown of air by increasing the phase velocity of the purtubing laser and vice versa. This is much noticable at higher energies probably due to absorption saturation. The FWHM of the refractive index with the highest input energy was found to be about $1.2 \mathrm{~mm}$. The higher the laser energy input, the higher the FWHM, indicating plasma expansion instead of increased heating.

For this work, $v_{p} \geq c$ and $v_{g} \leq c$ and these results show the behavior of the laser phase velocity in the plasma. The LIBS technique can, thus, be used to measure phase velocity changes in a plasma. The technique also gives valuable information about the electron collision frequency in the plasma. The plasma electron density and electron-neutral collision frequency are crucial in understanding the behavior of transport coefficients of plasmas [19]. Thus, a versatile and quick diagnostic tool (like LIBS) for $N_{e}$ and $v_{\mathrm{ec}}$ measurements could be very helpful in research application like ITER and JET tokamaks ${ }^{2}$ [24] and Plasma Focus Fusion devices [25], etc.

\section{Possible Applications of the LIBS Technique}

The LIBS detecting system can be modified and used to detect changes in electron concentration in the ionosphere due to earthquake activity and to detect infrared emissions from cracking

\footnotetext{
${ }^{2}$ ITER. [Online]. Available: http://www.iter.org/index.htm
} 
rocks, hours (or minutes) prior to an earthquake [26]. Recently, much study has been done to control lightning discharges both for energy storage and protection [27], [28]. Thus, if the competing dynamics of self-focusing, diffraction, and plasma defocusing are balanced, the self-guided laser beam can be propagated over multiple Rayleigh lengths. The profile of the refractive index and electron density in a plasma provides valuable information for designing plasma waveguides. To guide a Gaussian beam without distortion, one needs a concave parabolic-shaped electron density and a convex parabollic-shaped refractive index near the laser axis. The results of this paper show that the laser induced air plasma has such profiles.

\section{CONCLUSION}

Laser induced breakdown spectroscopy has been demonstrated to be a useful and versatile tool for density, phase velocity, plasma frequency, and refractive index measurements in an air plasma. The estimated FWHM of these profiles was found to be close to $1.2 \mathrm{~mm}$. The results confirm previous experimental results that the plasma counteracts higher energy deposits by expanding instead of getting hotter. The electron density at the center of the plasma plume is slightly hollow probably due to the effect of the shock wave. From the measured parameters, the behavior of plasma frequency and laser phase velocity change were determined. Laser phase velocity changes are due to a decrease in the real part of the refractive index, which in turn is caused by radial electron density gradients. The laser phase velocity then travels at a speed greater than the speed of light in the plasma. Understading this mechanism could find an application in lightning discharge control [29]. The laser induced air plasma has a concave parabollic-shaped electron density and a convex parabolic-shaped refractive index near the laser axis, i.e., around a diameter of $0.5 \mathrm{~mm}$. The electron density and electron-neutron collision frequency are crucial in understanding transport coefficients of large scale plasmas as found in thermonuclear fusion devices like tokamaks, etc.

\section{ACKNOWLEDGMENT}

The authors would like to express their gratefulness to the African Laser Center (ALC) and to the National Laser Center (NLC) for hosting M. Mathuthu during this research. They would also like to thank H. G. C. Human, H. van Wyk, and K. B. Mayo (of the NLC) for their technical support.

\section{REFERENCES}

[1] C. V. Bindhu, S. S. Harilal, M. S. Tillack, F. Najmabadi, and A. C. Gaeris, "Energy absorption and propagation in laser created sparks," Appl. Spectro., vol. 58, no. 6, pp. 719-726, Feb. 2004.

[2] X. C. Zeng, D. P. Singh, V. Palleschi, A. Salvetti, M. De Rosa, and M. Vaselli, "Simulation and experimental studies on the evolution of a laser spark in air," Laser Part. Beams, vol. 10, no. 4, pp. 707-713, Apr. 1992.

[3] M. Gatti, V. Palleschi, A. Salvetti, D. P. Singh, and M. Vaselli, "Sperical shock waves in laser produced plasmas in gas," Opt. Commun., vol. 69, no. 2, pp. 141-146, Dec. 1998.

[4] T. X. Phuoc, "An experimental and numerical study of laser-induced spark in air," Optics Lasers Eng., vol. 43, pp. 113-129, 2005.

[5] S. S. Harilal, B. O'Shay, S. Tillack, and M. V. Mathew, "Spectroscopic characterisation of laser induced tin plasma," J. Appl. Phys., vol. 98, pp. 1-7, July 2005.
[6] K. Dzierzega, K. Musiol, E. C. Benck, and J. R. Roberts, "Electron density meausrement in rf helium plasma by laser-collision indued fluorescence method," J. Appl. Phys., vol. 80, no. 6, pp. 3196-3201, Sep. 1996.

[7] A. Ciucci, M. Corsi, V. Palleschi, S. Rastelli, A. Salvetti, and E. Tognoni, "New procedure for quantitative elemental analysis by laser-induced plasma spectroscopy," Appl. Spectr., vol. 53, no. 8, pp. 960-964, Mar. 1999.

[8] M. Sabsabi, L. St-Onge, V. Detalle, and J. M. Lucas, Laser-induced breakdown spectroscopy: A new tool for precess control (2005). [Online]. Available: http://www.ndt.net/article/wcndt2004/html/htmltxt/ 679_sabsabi.htm

[9] A. Ciucci, V. Palleschi, S. Rastelli, R. Barbini, F. Colao, R. Fantoni, A. Palicci, S. Ribezzo, and H. J. L. van der Steen, "Trace pollutants analysis in soil by a time-resolved laser induced breakdown spectroscopy technique," Appl. Phys. B, vol. 63, pp. 185-190, Nov. 1996.

[10] G. Arca, A. Ciucci, V. Palleschi, S. Rastelli, and E. Tognoni, "Trace element analysis in water by the laser induced breakdown spectrodscopy technique," Appl. Spectr., vol. 51, no. 8, pp. 1102-1105, Mar. 1997.

[11] B. Y. Man, "Particle velocity, electron temperature and density profiles of pulsed laser induced plasmas in air at different ambient pressures," Appl. Phys. B, vol. 67, pp. 241-245, 1998.

[12] I. Borgia, L. M. F. Burgio, M. Corsi, R. Fantoni, V. Palleschi, A. Salvetti, M. C. Squarcialupi, and E. Tognoni, "Self-calibrated quantitative elemental analysis by laser induced plasma spectroscopy: application to pigment analysis," J. Cult. Heritage, vol. 1, pp. S281-S286, 2000.

[13] L. Bolognesi, M. Corsi, V. Palleschi, A. Salvetti, M. C. Squarcialupi, and E. Tognoni, "Calibration-free laser induced plasma spectroscopy for cultural heritage conservation and analysis," in Proc. 2nd Int. Congress Sci. Technol. Safeguard Cultural Heritage Mediterranean Basin, 2000, pp. 431-436.

[14] E. Tognoni, V. Palleschi, M. Corsi, and G. Cristotorettii, "Quantitative micro-analysis by laser induced breakdown spectroscopy: A review of the experimental approaches," Spectrochimica Acta Part B, vol. 57, pp. 1115-1130, Mar. 2002

[15] M. Villagran-Muniz, H. Sobral, and R. Navarro-Gonzalez, "Shock and thermal wave study of laser induced plasmas in air by the probe beam deflection technique," Meas. Sci. Technol., vol. 14, pp. 614-618, Mar. 2003.

[16] H. R. Griem, Plasma Spectroscopy. New York: McGraw-Hill, 1964.

[17] D. R. Lide, Handbook of Chemistry and Physics. Boca Raton, FL: CRC, 1990-1991.

[18] I. H. Hutchinson, Principles of Plasma Diagnostics, 2nd ed. Cambridge, U.K.: Cambridge Univ. Press, 2002.

[19] M. K. Howlader, Y. Yang, and J. R. Roth, "Time resolved measurement of electron number density and collision frequency for a fluorescent lamp plasma using microwave diagnostics," IEEE Trans. Plasma Sci., vol. 33, no. 3, pp. 1093-1099, Jun. 2005.

[20] A. Ciucci, V. Palleschi, S. Rastelli, A. Salvetti, D. P. Singh, and E. Tognoni, "Effect of imperfect focusing in laser induced plasma spectroscopy measurements," Il Nuovo Cimento, vol. 20 D, no. 10, pp. 1469-1478, Oct. 1998.

[21] B. Bastiaens, "Plasma channels with capillary discharges," Laser Phys Nonlinear Optics Group, Dept. Sci. Technol., Univ. Twente, 2005 [Online]. Available: http://lf.tnw.utwente.nl/project.php?projectid=10submenu $=16$

[22] S. I. Braginskii, Braginskii Equations (2005). [Online]. Available: http://farside.ph.utexas.edu/teaching/plasma/lectures/node35.html

[23] W. Demtroder, Laser Spectroscopy, 2nd enlarged ed. New York: Spinger-Verlag, 1996.

[24] R. S. Hemsworth and T. Inoue, "Positive and negative ion sources for magnetic fusion," IEEE Trans. Plasma Sci., vol. 33, no. 6, pp. 1799-1813, Dec. 2005.

[25] M. Mathuthu, T. G. Zengeni, and A. V. Gholap, "Design, fabrication and characterisation of a $2.3 \mathrm{~kJ}$ plasma focus device of negative polarity," Rev. Sci. Instrum., vol. 68, no. 3, pp. 1429-1432, 1997.

[26] "IEEE spectrum, earthquake alarm," IEEE Spectr., vol. 42, no. 12, pp. 16-21, Dec. 2005.

[27] P. Rambo, J. Schwartz, and D. Jean-Claude, "High voltage electrical discharges induced by an ultrshort pulse UV laser system," J. Opt. A: Appl. Opt., vol. 3, pp. 146-158, 2001.

[28] N. Khan, N. Mariun, I. Aris, and J. Yeak, "Laser triggered lightning discharge," New J. Phys., vol. 4, pp. 61.1-61.20, Aug. 2002.

[29] P. Mortensen, "Laser beam triggers lightning strike during Japanese experiment," Laser Focus World, Sep. 1997 [Online]. Available: http:// www.esdjournal.com/articles/lightn.htm 


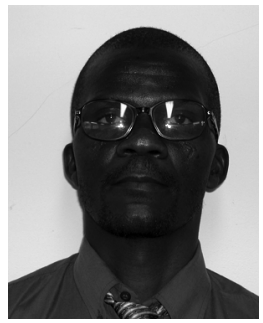

Manny Mathuthu (M'05) received the Ph.D. degree in plasma physics from the University of Zimbabwe, Harare, Zimbabwe, in 1999.

$\mathrm{He}$ is currently a Spectroscopy Physicist and a Lecturer at the University of Zimbabwe. Between 1994 and 1995, he was an Asian African Association for Plasma Training (AAAPT) Fellow at Quaid-I-Azam University, Pakistan. In 1997, he was also the AAAPT Fellow at Tshingwa University, Beijing, China.

Dr. Mathuthu was one of the recipients of the Third World Academy of Sciences (TWAS) Research Grant for the year 2001 and also one of the recipients of the Research Grant from the African Laser Center for 2005/2006.

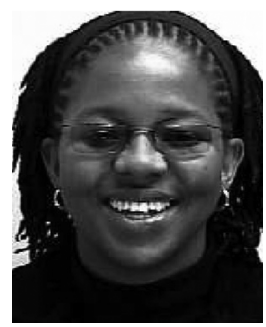

Rita M. Raseleka received the Doctorate in Technology degree in chemistry from Technikon Pretoria [now Tshwane University of Technology (TUT)], Pretoria, South Africa, in 2001.

She is currently a Lecturer at TUT, is a member of South African Chemical Institute, and a council member of the South African Council for Natural and Scientific Professionals. She did her postdoctorate studies at the National Laser Centre (NLC) of the Council for Scientific and Industrial Research (CSIR) between 2003 and 2004, which was funded by the National Research Foundation (NRF). She has co-authored nine scientific publications in international journals.

Dr. Raseleka is the NRF research grantholder from 2003 to date.

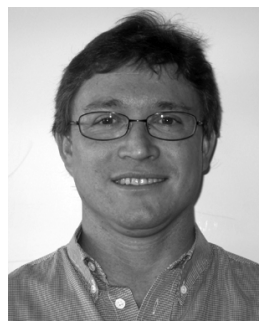

Andrew Forbes received the Ph.D. degree from the University of Natal, Durban, South Africa, in 1998.

He has spent several years working as an Applied Laser Physicist, first for the South African Atomic Energy Corporation on high power lasers for the MLIS process for Uranium, and then later on TEA $\mathrm{CO}_{2}$ lasers for laser unltrasonics. He is currently a Principal Researcher at the National Laser Center and works on beam propagation, wavefront control for laser beam shaping and laser resonator analysis and design. He is on the organizing committee of various local and international conferences and is the secretary of the South African Institute of Physics, Laser Optics and Spectroscopy subgroup. He has several publications in international journals.

Dr. Forbes is a member of SPIE and an honorary member of the University of Kwazulu-Natal, Durban, South Africa.

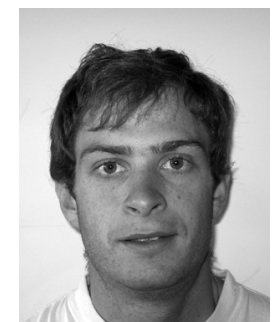

Nicholas West received the B.Sc. (Eng.) and M.Sc. (Eng.) degrees from the University of the Witwatersrand, Johannesburg, South Africa, in 2003 and 2004, respectively, where he is currently doing research for his doctoral thesis on long gap laser-induced breakdown.

His research interests include the fields of high voltage engineering, lightning research, and laser-induced plasma phenomena. 